\title{
Correlated two-nucleon stripping reactions
}

\author{
J. A. Tostevin* and G. Podolyák \\ Department of Physics, School of Electronics and Physical Sciences, University of Surrey, Guildford, Surrey GU2 7XH, \\ United Kingdom \\ B. A. Brown and P. G. Hansen \\ National Superconducting Cyclotron Laboratory, Michigan State University, East Lansing, Michigan 48824, USA \\ and Department of Physics and Astronomy, Michigan State University, East Lansing, Michigan 48824, USA
}

(Received 6 August 2004; published 9 December 2004)

\begin{abstract}
The cross sections for the stripping of two correlated nucleons from light and medium-mass nuclei are considered. Such reactions are of interest both as a means for populating and identifying low-lying excited states of very exotic nuclear species and as a potential direct spectroscopic probe of two-nucleon correlations in such systems. A calculation scheme that combines the full shell model two-nucleon spectroscopic amplitudes with eikonal reaction theory is presented. The theoretical predictions of the method, and of more approximate schemes, are compared with new data on two-proton removal from ${ }^{28} \mathrm{Mg}$. The combined full shell model structure amplitudes and reaction dynamics predictions are in good agreement with the available measurements. First indications of the sensitivity of the reaction mechanism to the spatial and angular momentum structure of the stripped two-nucleon wave functions are also discussed and clarified.
\end{abstract}

DOI: 10.1103/PhysRevC.70.064602

PACS number(s): 21.10.Jx, 24.10.-i, 25.60.Gc, 27.30.+t

\section{INTRODUCTION}

Single-nucleon knockout reactions, either with [1-4] or without [5-7] coincident gamma-ray detection, have now been the subject of numerous systematic studies. In particular, since their first exploratory application to the phosphorus isotopes, in [1], single-nucleon knockout experiments with gamma-ray detection capability have been extensively tested and are being exploited to study the single-nucleon spectroscopy of exotic light [4,8-13] and more medium-mass nuclei [14-16]. They are currently being used to study both the relative and absolute single-nucleon spectroscopy of neutron- and proton-rich nuclei [10,14-17]. Recent reviews of the theoretical basis and the experimental status of such studies can be found in Refs. [2,3,13].

Single-nucleon knockout reactions using intermediateenergy exotic beams, and carried out in inverse kinematics, are fast, peripheral reactions. The residual nucleus, having lost one nucleon via the diffractive dissociation (elastic breakup) or stripping (target absorption) mechanisms on a thick, light nuclear target, is then detected in the forward direction with a velocity close to that of the particles of the incident beam. The technique has now been demonstrated to be remarkably sensitive [3]. Analyses of these data using eikonal few-body reaction theory have been shown to yield results of good accuracy [7,18-20] and to offer formal, practical, and quantitative advantages over alternative direct reaction approaches [13]. This is allowing the possibility to systematically probe aspects of effective interaction theories and correlation effects on both neutron and proton orbitals underlying the shell model.

The generalization of these techniques to two-nucleon knockout reactions and the magnitude of the associated cross

*Electronic address: j.tostevin@surrey.ac.uk sections are of interest for two reasons. The first is the potential to use two-nucleon knockout as a means for populating and identifying the ground and low-lying excited states of exotic, asymmetric nuclei. The second is the potential to use the reaction, in addition, as a spectroscopic probe of two-nucleon correlations in such exotic systems and hence to assess modern nuclear structure calculations of these effects. It has recently been proposed that two-proton removal reactions from nuclei on the neutron-rich side of the valley of stability, at high energy, do proceed as direct processes. The separation energies and nucleon thresholds in such systems suggest very strongly that direct two-proton removal will be the only significant path to bound, $Z-2$ residue final states. Compelling experimental evidence was offered by both the measured inclusive cross section and the parallel momentum distribution of the reaction residues in two-proton knockout from ${ }^{28} \mathrm{Mg}$ [21].

Unlike single-nucleon transfer and knockout reaction spectroscopy, two-nucleon removal reaction theories do not factorize naturally into a structural (spectroscopic) factor and a dynamical single-particle cross section. The reaction dynamics and structure are now more intimately coupled and the reaction amplitudes are, in general, a coherent linear superposition of many contributing two-nucleon configuration terms, e.g., [22]. In this paper we present an original, eikonal model scheme for the calculation of the stripping (inelastic breakup) component of the two-nucleon removal reaction. This approach is able to combine the two-nucleon spectroscopic amplitudes from modern shell model calculations with the appropriate generalization of few-body eikonalbased reaction theory, as has been discussed extensively for one-nucleon knockout $[3,13]$.

The necessary formal developments are presented in Sec. II. In Sec. III the theoretical predictions of the model are compared both with more approximate calculations and with the results of new measurements of the two-proton knockout 


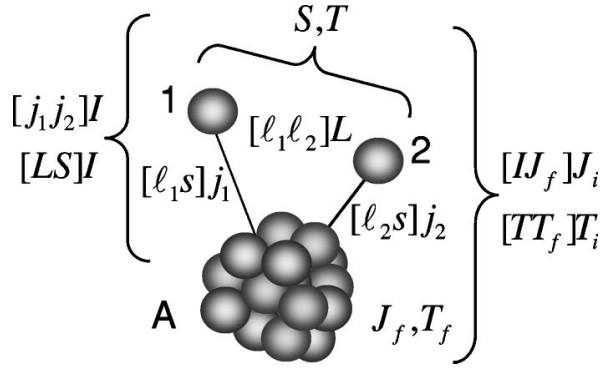

FIG. 1. Schematic of the angular momentum couplings used in the description of the two-nucleon knockout reaction.

reaction from the neutron-rich nucleus ${ }^{28} \mathrm{Mg}$ [21]. The twonucleon spin-correlation selectivity of the reaction mechanism is considered in Sec. IV and a second application, to two-neutron knockout from three neutron-deficient nuclei, is considered briefly in Sec. V.

\section{FORMALISM}

Here we consider two-nucleon knockout from a secondary projectile beam at intermediate energy. We consider the projectile as an antisymmetrized $(A+2)$-nucleon system and denote its many-body wave function by $\Psi(A, 1,2)$. We do not enumerate explicitly all nucleon coordinates. Thus $\Psi(A, 1,2)$ represents the initial, $(A+2)$-nucleon (shell model) ground state of the nuclei of the beam. These are assumed to carry total angular momentum and isospin $J_{i}$ and $T_{i}$ with projections $M_{i}$ and $\tau_{i}$. Following the (assumed sudden) removal of two nucleons in a peripheral, high-speed collision with the target, the final, antisymmetrized $A$-body residual (or core) nucleus will, in general, be found in one of a number of final states $\Phi(A)$, with spin and isospin $J_{f}, T_{f}$ and projections $M_{f}, \tau_{f}$.

\section{A. Two-nucleon amplitudes}

Quite generally, the two removed nucleons, denoted 1 and 2 , must be assumed to be stripped from a set of one or more active (and partially occupied) single-particle orbitals $\phi_{j}$. These have spherical (shell model) single-particle quantum numbers $n(\ell s) j, m$. The isospin and angular momentum couplings involved are summarized in Fig. 1, where the two active, removed nucleons will be assumed to couple to an intermediate total angular momentum $I, \mu$ and total isospin $T, \tau$.

The shell model two-nucleon overlap functions of these two nucleons in the projectile ground state, relative to a specified residue or core state $f$, is then a coherent sum over all the possible contributing two-particle configurations, as

$$
\begin{aligned}
\Psi_{J_{i} M_{i}}^{(f)}(1,2) & \equiv\left\langle\Phi_{J_{f} M_{f}}(A) \mid \Psi_{J_{i} M_{i}}(A, 1,2)\right\rangle \\
& =\sum_{I \mu \alpha} C_{\alpha}^{J_{i} J_{f} I}\left(I \mu J_{f} M_{f} \mid J_{i} M_{i}\right)\left[\overline{\phi_{j_{1}}(1) \otimes \phi_{j_{2}}(2)}\right]_{I \mu},
\end{aligned}
$$

where $\alpha \equiv\left\{n_{1} \ell_{1} j_{1}, n_{2} \ell_{2} j_{2}\right\}$ denotes each of the available orbital pairs which contribute. In this equation

$$
\begin{aligned}
{\left[\overline{\phi_{j_{1}}(1) \otimes \phi_{j_{2}}(2)}\right]_{I \mu}=} & -N_{12}\left\langle 1,2\left|\left[a_{j_{1}}^{\dagger} \otimes a_{j_{2}}^{\dagger}\right]_{I \mu}\right| 0\right\rangle \\
= & D_{\alpha} \sum_{m_{1} m_{2}}\left(j_{1} m_{1} j_{2} m_{2} \mid I \mu\right)\left[\phi_{j_{1}}^{m_{1}}(1) \phi_{j_{2}}^{m_{2}}(2)\right. \\
& \left.-\phi_{j_{1}}^{m_{1}}(2) \phi_{j_{2}}^{m_{2}}(1)\right]
\end{aligned}
$$

is a normalized, antisymmetrized nucleon-pair wave function and $D_{\alpha}=N_{12} / \sqrt{2}=1 / \sqrt{2\left(1+\delta_{12}\right)}$. So as not to complicate the notation we will not yet show the isospin labels and coupling explicitly. We will only finally include these into the formalism for completeness. The $C_{\alpha}^{j_{\alpha} J_{f} I}$ in Eq. (1) are the signed two-nucleon amplitudes which carry the structure calculation details; in particular, the information on the parentage and phase of each of the participating two-nucleon configurations in the projectile ground state with respect to the final states $f$ of the residue.

\section{B. Eikonal model of two-nucleon stripping}

We will show, following [21], that two-nucleon removal reactions, from exotic nuclei having even a modest asymmetry (isospin) with respect to stable species, are expected to proceed essentially as a direct reaction process. We will later examine in detail the case of two-proton removal from ${ }^{28} \mathrm{Mg}$, only two neutrons away from stable ${ }^{26} \mathrm{Mg}$, as a particular example of this direct reaction mechanism. The direct, twonucleon knockout reaction mechanism is thus expected to be applicable to studies of a large region of the nuclear chart.

We will calculate the dominant stripping contribution to the two-nucleon removal cross section. This is the projectile ground state average,

$\sigma_{s t r}=\frac{1}{2 J_{i}+1} \sum_{M_{i}} \int d \vec{b}\left\langle\left.\Psi_{J_{i} M_{i}}|| \mathcal{S}_{f}\right|^{2}\left(1-\left|\mathcal{S}_{1}\right|^{2}\right)\left(1-\left|\mathcal{S}_{2}\right|^{2}\right) \mid \Psi_{J_{i} M_{i}}\right\rangle$,

and an integral over all projectile center-of-mass (c.m.) impact parameters $b$. Here the $\mathcal{S}_{i}$ are the eikonal $S$ matrices [18] for the scattering of the two nucleons $(1,2)$ and of the $A$-body residue $f$ from the target. Each is a function of the impact parameter of that constituent. These $\mathcal{S}_{i}$ will be assumed to be spin independent. This cross section expression reflects the stripping (inelastic breakup) mechanism in which the residue interacts at most elastically with the target, survives the collision, and escapes to infinity; reflected by $\left|\mathcal{S}_{f}\right|^{2}$. The two removed nucleons interact inelastically with the target and are absorbed from the elastic channel; as described by their absorption probabilities $\left(1-\left|\mathcal{S}_{1}\right|^{2}\right)$ and $\left(1-\left|\mathcal{S}_{2}\right|^{2}\right)$.

We make a small number of quite reasonable, but simplifying approximations. We first assume that the residue-target $S$ matrix is diagonal with respect to different final states $f$ of the residue, and that this diagonal interaction is the same as that for the residue ground state (denoted $=\mathcal{S}_{c}$ ) for all final states $f$. This has been termed the spectator-core approximation when used in single-nucleon knockout [23]. It assumes that the amplitudes for dynamical excitation of the core during the collision are small.

We also neglect explicit recoil effects associated with the heavy mass $A$ residue. It follows that 


$$
\left\langle\left.\Phi_{J_{f}^{\prime} M_{f}^{\prime}}(A)|| \mathcal{S}_{f}\right|^{2} \mid \Phi_{J_{f} M_{f}}(A)\right\rangle=\left|\mathcal{S}_{c}(b)\right|^{2} \delta_{f f^{\prime}} \delta_{J_{f} J_{f}^{\prime}} \delta_{M_{f} M_{f}^{\prime}},
$$

with $b$ the projectile c.m. impact parameter. As we also consider here only nucleon knockout from deeply bound singleparticle states, we do not calculate other possible contributions to the two-nucleon removal cross section, and which we assume are small. These involve diffraction dissociation processes in which one or both nucleons are dissociated from the projectile by their elastic collisions with the target or, in the case of one nucleon being dissociated, the second being absorbed.

Having made the spectator-core and the no-recoil approximation, the inclusive stripping cross section is then the incoherent sum of the contributions from each residue final state and so, with $\hat{J}^{2}=(2 J+1)$, is

$$
\begin{aligned}
\sigma_{s t r}=\sum_{f} \sigma_{s t r}^{(f)}= & \sum_{f}\left[\int d \vec{b}\left|\mathcal{S}_{c}\right|^{2} \frac{1}{\hat{J}_{i}^{2} \sum_{M_{i}}}\left\langle\Psi_{J_{i} M_{i}}^{(f)}\right|\left(1-\left|\mathcal{S}_{1}\right|^{2}\right)\right. \\
& \left.\times\left(1-\left|\mathcal{S}_{2}\right|^{2}\right) \mid \Psi_{J_{i} M_{i}}^{(f)}\right] .
\end{aligned}
$$

Here the bra-ket denotes integration over the spatial coordi- nates of the two removed nucleons, $\vec{r}_{1}$ and $\vec{r}_{2}$, and the integration over all spin variables, denoted by

$$
\left\langle\Psi_{J_{i} M_{i}}^{(f)}|\cdots| \Psi_{J_{i} M_{i}}^{(f)}\right\rangle=\int d \vec{r}_{1} \int d \vec{r}_{2}\left\langle\Psi_{J_{i} M_{i}}^{(f)}|\cdots| \Psi_{J_{i} M_{i}}^{(f)}\right\rangle_{s p}
$$

Since all the particle-target $S$ matrices are assumed spin independent, we require only the spin average of the twonucleon wave functions, that is,

$$
\begin{aligned}
\frac{1}{\hat{J}_{i}^{2}} \sum_{M_{i}}\left\langle\Psi_{J_{i} M_{i}}^{(f)} \mid \Psi_{J_{i} M_{i}}^{(f)}\right\rangle_{s p} \\
=\frac{1}{\hat{J}_{i}^{2}} \sum_{M_{f} M_{i} I \mu \alpha I^{\prime} \mu^{\prime} \alpha^{\prime}} C_{\alpha^{\prime}}^{J_{j^{\prime}} I^{\prime} I^{\prime}} C_{\alpha}^{J_{i} J_{f} I^{\prime}} \\
\quad \times\left(I^{\prime} \mu^{\prime} J_{f} M_{f} \mid J_{i} M_{i}\right)\left(I \mu J_{f} M_{f} \mid J_{i} M_{i}\right) \\
\quad \times\left\langle\left[\overline{\phi_{j_{1}^{\prime}}(1) \otimes \phi_{j_{2}^{\prime}}(2)}\right]_{I^{\prime} \mu^{\prime}} \mid\left[\overline{\phi_{j_{1}}(1) \otimes \phi_{j_{2}}(2)}\right]_{I \mu}\right\rangle_{s p} .
\end{aligned}
$$

Upon using Eqs. (1) and (2) this reduces to

$$
\begin{aligned}
\frac{1}{\hat{J}_{i}^{2}} \sum_{M_{i}}\left\langle\Psi_{J_{i} M_{i}}^{(f)} \mid \Psi_{J_{i} M_{i}}^{(f)}\right\rangle_{s p}= & \sum_{I \alpha \alpha^{\prime}} 2 D_{\alpha} D_{\alpha^{\prime}} \frac{C_{\alpha^{\prime}}^{J_{j} J_{f}^{I} C_{\alpha}^{J_{i} J_{f} I}}}{\hat{I}^{2}} \sum_{m_{1} m_{2} m_{1}^{\prime} m_{2}^{\prime} \mu}\left(j_{1} m_{1} j_{2} m_{2} \mid I \mu\right)\left(j_{1}^{\prime} m_{1}^{\prime} j_{2}^{\prime} m_{2}^{\prime} \mid I \mu\right) \\
& \times\left[\left\langle\phi_{j_{1}^{\prime}}^{m_{1}^{\prime}} \mid \phi_{j_{1}}^{m_{1}}\right\rangle_{s p}\left\langle\phi_{j_{2}^{\prime}}^{m_{2}^{\prime}} \mid \phi_{j_{2}}^{m_{2}}\right\rangle_{s p}-\left\langle\phi_{j_{1}^{\prime}}^{m_{1}^{\prime}} \mid \phi_{j_{2}}^{m_{2}}\right\rangle_{s p}\left\langle\phi_{j_{2}^{\prime}}^{m_{2}^{\prime}} \mid \phi_{j_{1}}^{m_{1}}\right\rangle_{s p}\right] .
\end{aligned}
$$

We will refer to terms from the first product in the last bracket as being direct and terms from the second product as exchange. The general form of this spin average for each single-particle state (with the nucleon spin $s=1 / 2$ understood) has the following multipole expansion [13]:

$$
\begin{aligned}
\left\langle\phi_{j^{\prime}}^{m^{\prime}} \mid \phi_{j}^{m}\right\rangle_{s p}= & \sum_{k q}\left(j^{\prime} m^{\prime} k q \mid j m\right)\left[\frac{\hat{\ell} \hat{\ell}^{\prime} \hat{j}^{\prime}}{\sqrt{4 \pi}}(-1)^{2 s+j+j^{\prime}-\ell}\left(\ell 0 \ell^{\prime} 0 \mid k 0\right)\right. \\
& \left.\times W\left(j s k \ell^{\prime} ; \ell j^{\prime}\right) u_{j^{\prime} \ell^{\prime}}(r) u_{j \ell}(r) Y_{k q}(\hat{\vec{r}})\right] \\
\equiv & \sum_{k q}\left(j^{\prime} m^{\prime} k q \mid j m\right)\left\langle\left\langle j^{\prime} \ell^{\prime}\left|\mathcal{O}_{k q}(\vec{r})\right| j \ell\right\rangle\right\rangle
\end{aligned}
$$

where the $u_{j \ell}(r)$ are the single-particle radial wave functions and $\langle\langle\cdots\rangle\rangle$ is used as shorthand for the square-bracketed expression. These single-particle spin averages actually enter the stripping calculation as a product with their corresponding nucleonic absorption factors $\left(1-\left|\mathcal{S}_{i}\right|^{2}\right)$ and are integrated over the appropriate single-particle position coordinate. Explicitly,

$$
\begin{aligned}
\int d \vec{r}\left(1-|\mathcal{S}|^{2}\right)\left\langle\phi_{j^{\prime}}^{m^{\prime}} \mid \phi_{j}^{m}\right\rangle_{s p}= & \sum_{k q}\left(j^{\prime} m^{\prime} k q \mid j m\right) \int d \vec{r}\left(1-|\mathcal{S}|^{2}\right) \\
& \times\left\langle\left\langle j^{\prime} \ell^{\prime}\left|\mathcal{O}_{k q}(\vec{r})\right| j \ell\right\rangle\right\rangle \\
\equiv & \sum_{k q}\left(j^{\prime} m^{\prime} k q \mid j m\right)\left\{j^{\prime} \ell^{\prime}\left|\mathcal{F}_{k q}(b)\right| j \ell\right\},
\end{aligned}
$$

which defines the brackets $\left\{j^{\prime} \ell^{\prime}\left|\mathcal{F}_{k q}(b)\right| j \ell\right\}$ that are now functions only of the angular momenta indicated and the projectile $\mathrm{cm}$ impact parameter $b$. Equations (10) are computed (at each $b$ ) by numerical quadratures over the cylindrical coordinates of $\vec{r} \equiv\left(\vec{b}_{r}, z\right)=\left(b_{r}, \phi_{r}, z\right)$, measured relative to $\vec{b}$. The integral of $\left\langle\left\langle j^{\prime} \ell^{\prime}\left|\mathcal{O}_{k q}(\vec{r})\right| j \ell\right\rangle\right\rangle$ over $z$ can be precalculated since the terms involving $\mathcal{S}\left(\left|\vec{b}+\vec{b}_{r}\right|\right)$ are $z$ independent. 
Upon simplifying the remainder of the angular momentum coupling coefficients we can write

$$
\begin{gathered}
\frac{1}{\hat{J}_{i}^{2}} \sum_{M_{i}}\left\langle\Psi_{J_{i} M_{i}}^{(f)}\left|\left(1-\left|\mathcal{S}_{1}\right|^{2}\right)\left(1-\left|\mathcal{S}_{2}\right|^{2}\right)\right| \Psi_{J_{i} M_{i}}^{(f)}\right\rangle \\
=\sum_{\alpha \alpha^{\prime} I} 2 D_{\alpha} D_{\alpha^{\prime}} C_{\alpha^{\prime}}^{J_{i} J_{f} I} C_{\alpha}^{J_{i} J_{f} I} \hat{j}_{1} \hat{j}_{2} \\
\quad \times \sum_{K Q} \frac{(-)^{Q}}{\hat{K}^{2}}[\text { direct - exchange }]
\end{gathered}
$$

and where

$$
\begin{aligned}
\text { direct } \equiv & (-)^{I-j_{1}-j_{2}^{\prime}} W\left(j_{1} j_{1}^{\prime} j_{2} j_{2}^{\prime} ; K I\right)\left\{j_{1}^{\prime} \ell_{1}^{\prime}\left|\mathcal{F}_{K-Q}(b)\right| j_{1} \ell_{1}\right\} \\
& \times\left\{j_{2}^{\prime} \ell_{2}^{\prime}\left|\mathcal{F}_{K Q}(b)\right| j_{2} \ell_{2}\right\},
\end{aligned}
$$

exchange $\equiv(-)^{j_{2}^{\prime}-j_{1}} W\left(j_{1} j_{2}^{\prime} j_{2} j_{1}^{\prime} ; K I\right)\left\{j_{2}^{\prime} \ell_{2}^{\prime}\left|\mathcal{F}_{K-Q}(b)\right| j_{1} \ell_{1}\right\}$

$$
\times\left\{j_{1}^{\prime} \ell_{1}^{\prime}\left|\mathcal{F}_{K Q}(b)\right| j_{2} \ell_{2}\right\} .
$$

Referring back to Eq. (5), we note that the stripping cross section $\sigma_{s t r}^{(f)}$, to a given residue final state $f$, with angular momentum $J_{f}$, is now calculated using Eq. (11), since

$$
\begin{aligned}
\sigma_{s t r}^{(f)}= & 2 \pi \int d b b\left|\mathcal{S}_{c}\right|^{2}\left\{\frac{1}{\hat{J}_{i}^{2}} \sum_{M_{i}}\left\langle\Psi_{J_{i} M_{i}}^{(f)}\right|\left(1-\left|\mathcal{S}_{1}\right|^{2}\right)\right. \\
& \left.\times\left(1-\left|\mathcal{S}_{2}\right|^{2}\right)\left|\Psi_{J_{i} M_{i}}^{(f)}\right\rangle\right\} .
\end{aligned}
$$

\section{Isospin dependence}

The inclusion of isospin labels in Eq. (1) and the subsequent equations leads to rather simple modifications. Equation (1) becomes

$$
\begin{aligned}
\Psi_{J_{i} M_{i} T_{i} \tau_{i}}^{(f)}(1,2) \equiv & \left\langle\Phi_{J_{f} M_{f} T_{f} \tau_{f}}(A) \mid \Psi_{J_{i} M_{i} T_{i} \tau_{i}}(A, 1,2)\right\rangle \\
= & \sum_{I \mu \alpha T \tau} C_{\alpha}^{J_{i} J_{f} I T_{i} T_{f} T}\left(I \mu J_{f} M_{f} \mid J_{i} M_{i}\right)\left(T \tau T_{f} \tau_{f} \mid T_{i} \tau_{i}\right) \\
& \times\left[\overline{\phi_{j_{1}}(1) \otimes \phi_{j_{2}}(2)}\right]_{I \mu}^{T \tau}
\end{aligned}
$$

where now

$$
\begin{aligned}
{\left[\overline{\phi_{j_{1}}(1) \otimes \phi_{j_{2}}(2)}\right]_{I \mu}^{T \tau}=} & D_{\alpha} \sum_{m_{1} m_{2}}\left(j_{1} m_{1} j_{2} m_{2} \mid I \mu\right)\left[\phi_{j_{1}}^{m_{1}}(1) \phi_{j_{2}}^{m_{2}}(2)\right. \\
& \left.-(-)^{1+T} \phi_{j_{1}}^{m_{1}}(2) \phi_{j_{2}}^{m_{2}}(1)\right] \chi_{T \tau}(1,2) .
\end{aligned}
$$

Thus, isospin introduces an additional phase factor of $(-)^{1+T}$ in front of the exchange term in Eq. (11), in which equation the two-nucleon amplitudes $C$ are now also dependent on $T$. In addition, the final expression for the stripping cross section, Eq. (14), must be multiplied by the square of the usual overall isospin coupling Clebsh-Gordan coefficient $\left(T \tau T_{f} \tau_{f} \mid T_{i} \tau_{i}\right)$.

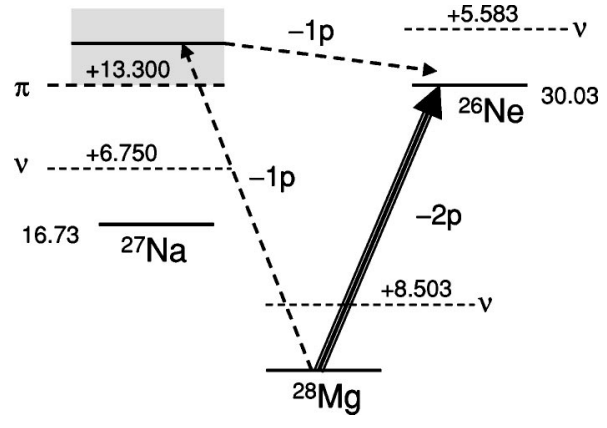

FIG. 2. Energy diagram of the neutron-rich $N=16$ isotones ${ }^{28} \mathrm{Mg},{ }^{27} \mathrm{Na}$, and ${ }^{26} \mathrm{Ne}$, showing the single-neutron $(\nu)$ and proton $(\pi)$ separation energies for each nucleus. The diagram shows that nondirect population of the bound states of ${ }^{26} \mathrm{Ne}$, by one-proton removal to excited ${ }^{27} \mathrm{Na}$ followed by proton evaporation, would involve states high above the (much lower) neutron evaporation threshold and so is expected to be negligible.

\section{APPLICATION TO TWO-PROTON KNOCKOUT FROM ${ }^{28} \mathrm{Mg}$}

It has recently been proposed that two-proton removal from a neutron-rich system at high energy proceeds as a direct reaction [21]. The evidence was offered by both the measured inclusive cross section of the ${ }^{9} \mathrm{Be}\left({ }^{28} \mathrm{Mg},{ }^{26} \mathrm{Ne}\right) X$ reaction and also by the parallel momentum distribution of the reaction residues. The energetics of the $N=16$ isotones, shown schematically in Fig. 2, also suggest strongly that direct two-proton $(-2 p)$ removal is the only expected route to the observed bound ${ }^{26} \mathrm{Ne}$ final states. Different approximations to the treatment of the structure of ${ }^{28} \mathrm{Mg}$, within the eikonal reaction theory, were also considered in Ref. [21]. We are now in a position to discuss and elaborate upon these. We concentrate, however, on the quantitative description of the integrated and partial knockout cross sections.

Specifically, we consider the knockout of two protons from ${ }^{28} \mathrm{Mg}\left(0^{+}\right)$at $82.3 \mathrm{MeV} /$ nucleon incident energy and assume, consistent with data, that final states will be populated with ${ }^{26} \mathrm{Ne}\left(J^{\pi}\right)$ residues in the $0^{+}$ground state and the $2^{+}(2.02 \mathrm{MeV}), 4^{+}(3.50 \mathrm{MeV})$, and second $2^{+}(3.70 \mathrm{MeV})$ excited states $[21,24-26]$. The theoretical excited state energies from the shell model are in precise agreement with experiment. The measured cross sections to the four final states, $\sigma_{\text {expt }}\left(J_{f}^{\pi}\right)$, are collected in Table I.

The $S$ matrices in Eq. (14) are calculated from assumed core and target one-body matter densities using the optical limit of Glauber theory [13,27]. A Gaussian nucleon-nucleon (NN) effective interaction is assumed [11] with a range of $0.5 \mathrm{fm}$. This calculates residue- and nucleon-target $S$ matrices and corresponding reaction cross sections in line with measurements in the $50-100 \mathrm{MeV} /$ nucleon energy range, e.g., [28]. The strength of the interaction is determined, in the usual way [29], by the free $p p$ and $n p$ cross sections and the real-to-imaginary ratios of the forward NN scattering amplitudes, $\alpha_{p p}$ and $\alpha_{n p}$. The latter are, however, of no consequence for the calculation of the stripping term under discussion, which is determined by the $\left|\mathcal{S}_{i}\right|^{2}$. Densities of the target and the core were also assumed to have Gaussian shapes 
TABLE I. Calculated and experimental cross sections and deduced effective spectroscopic factors for two-proton knockout from ${ }^{28} \mathrm{Mg}$ at $82.3 \mathrm{MeV} /$ nucleon. The theoretical spectroscopic factors are calculated in the uncorrelated approximation $S_{u n c}$ and when including the full shell model two-proton amplitudes, $S_{t h}$. $S_{t h}$ and $S_{\text {expt }}$ are computed relative to the unit cross section for removal of an uncorrelated $0 d$ proton pair, $\sigma_{22}=0.29 \mathrm{mb}$

\begin{tabular}{cccccc}
\hline \hline$J_{f}^{\pi}$ & $S_{\text {unc }}$ & $S_{\text {expt }}$ & $\sigma_{\text {expt }}(\mathrm{mb})$ & $S_{\text {th }}$ & $\sigma_{\text {th }}(\mathrm{mb})$ \\
\hline $0^{+}$ & 1.33 & $2.4(5)$ & $0.70(15)$ & 1.83 & 0.532 \\
$2^{+}$ & 1.67 & $0.3(5)$ & $0.09(15)$ & 0.54 & 0.157 \\
$4^{+}$ & 3.00 & $2.0(3)$ & $0.58(9)$ & 1.79 & 0.518 \\
$2_{2}^{+}$ & - & $0.5(3)$ & $0.15(9)$ & 0.78 & 0.225 \\
Sums & 6 & $5.2(4)$ & $1.50(10)$ & 4.94 & 1.43 \\
\hline \hline
\end{tabular}

with root mean squared (rms) matter radii of 2.36 for ${ }^{9} \mathrm{Be}$ and $2.90 \mathrm{fm}$ for ${ }^{26} \mathrm{Ne}$ [30]. Extensive calculations for onenucleon knockout have shown the detailed radial form of the density to be unimportant, e.g., [16].

In our complete calculations, the shell model dictates that the removed protons are stripped from three active orbitals, the $0 d_{5 / 2}, 0 d_{3 / 2}$, and $1 s_{1 / 2}$ states. The corresponding spectroscopic coefficients $C_{\alpha}^{J_{i} J_{f} I}$ were calculated with the code OXBASH [31] in the $s d$-shell model space with the USD Hamiltonian [32]. These are provided in the TNA (twonucleon amplitude) output files from OXBASH. The relative phases of the amplitudes, which will be presented explicitly in Table II for ${ }^{28} \mathrm{Mg}$, are based on radial wave functions $u_{j \ell}$ that are positive near the origin, assuming the $(\ell s) j$ angular momentum coupling scheme. All radial wave functions are real, and do not contain an $i^{\ell}$ factor used by some authors.

Whenever needed, these single-particle wave functions $u_{j \ell}(r)$ are calculated in a Woods-Saxon potential well with conventional radius and diffuseness parameters, $r_{0}=1.25 \mathrm{fm}$ and $a=0.70 \mathrm{fm}$, respectively. The strength of the binding potential is adjusted to reproduce the physical separation energy. The experimental two proton separation energy is $S_{2 p}$ $=30.03 \mathrm{MeV}$. No spin-orbit potential is included and thus the $0 d_{5 / 2}$ and $0 d_{3 / 2}$ states are identical. Also, due to the large separation energy, we have not included the small corrections to the nucleon separation energies for the energy differences of the excited final states.

\section{A. Role of correlations}

In addition to the fully correlated scheme developed in this paper, we will consider briefly the following, more ap- proximate, prescriptions discussed in [21]. These were as follows. (1) To consider that the four valence protons in ${ }^{28} \mathrm{Mg}$ are restricted to a $\left[0 d_{5 / 2}\right]^{4}$ subshell configuration, but that they are otherwise uncorrelated. (2) To consider the relative strength of the final state populations, $S_{r e l}$, based on the components of the full shell model wave functions with the two nucleons having spin $S=0$ and an $s$ state of relative motion: as would be sampled, for instance, in the $(p, t)$ and $\left({ }^{3} \mathrm{He}, n\right)$ two-nucleon transfer vertices [33]. Here we will extend the latter to calculate exactly, and absolutely, that part of the two-nucleon stripping cross section arising from configurations with $S=0$ and $T=1$. In this way we can obtain a measure of the extent to which both spin-singlet and spintriplet pairs are sampled within the knockout mechanism.

\section{B. Uncorrelated stripping}

If the two removed nucleons are assumed to be uncorrelated, other than being bound to the same center, then the cross section for removal of the nucleons from the pair of orbitals $\ell_{1}$ and $\ell_{2}$ is, neglecting spin-orbit interactions,

$$
\sigma_{\ell_{1} \ell_{2}}=\int d \vec{b}\left|\mathcal{S}_{c}\right|^{2} \prod_{i=1,2} \frac{1}{2 \ell_{i}+1} \sum_{m_{i}}\left\langle\ell_{i} m_{i}\left|\left(1-\left|\mathcal{S}_{i}\right|^{2}\right)\right| \ell_{i} m_{i}\right\rangle .
$$

Assuming therefore that the valence proton structure in ${ }^{28} \mathrm{Mg}$ is $\left[0 d_{5 / 2}\right]^{4}$, several results follow. The first is that the calculated (unit) cross section for removal of a $\left[0 d_{5 / 2}\right]^{2}$ pair is, given the model parameters, $\sigma_{22}=0.29 \mathrm{mb}$. This sets the scale for the anticipated cross section. Based on an assumed $\left[0 d_{5 / 2}\right]^{n}$ ground state (with $n=4$ for ${ }^{28} \mathrm{Mg}$ ) this predicts an integrated cross section of $n(n-1) \sigma_{22} / 2$, or $1.8 \mathrm{mb}$, in reasonable agreement with the measured inclusive value of $1.50(10) \mathrm{mb}$ in Table I. However, it also follows in this uncorrelated limit that this cross section yield [and associated spectroscopic strength $S_{u n c}\left(J_{f}^{\pi}\right)$ ], for removal of a pair from a $0^{+},[j]^{n}$ occupied subshell, will be spread between final states $J_{f}^{\pi}$, determined by the corresponding coefficients of fractional parentage $\left(\left(j^{n-2}\right) v J_{f},\left(j^{2}\right) J_{f} \mid\left(j^{n}\right) 0\right)$ where $v$ is the seniority of the state. Explicitly, we have [22]

$$
S_{u n c}\left(J_{f}^{\pi}=0^{+}\right)=\frac{n(n-1)}{2}\left[\frac{2 j+3-n}{(n-1)(2 j+1)}\right],
$$

TABLE II. The $s d$-shell model two-nucleon spectroscopic amplitudes $C_{\alpha}^{J_{j} J_{f} I}\left(\equiv C_{\alpha}^{0 J_{f} J_{f}}\right)$ for the required ${ }^{28} \mathrm{Mg}\left(0^{+}\right) \rightarrow{ }^{26} \mathrm{Ne}\left(J_{f}^{\pi}\right)$ two-proton removal transitions. The assumed phase conventions are discussed in the text.

\begin{tabular}{cccccccc}
\hline \hline$J_{f}^{\pi}$ & $E^{*}(\mathrm{MeV})$ & {$\left[0 d_{3 / 2}\right]^{2}$} & {$\left[0 d_{3 / 2} 0 d_{5 / 2}\right]$} & {$\left[0 d_{5 / 2}\right]^{2}$} & {$\left[1 s_{1 / 2} 0 d_{3 / 2}\right]$} & {$\left[1 s_{1 / 2} 0 d_{5 / 2}\right]$} & {$\left[1 s_{1 / 2}\right]^{2}$} \\
\hline $0_{1}^{+}$ & 0.0 & -0.30146 & - & -1.04685 & - & - & -0.30496 \\
$2_{1}^{+}$ & 2.02 & -0.05030 & 0.37358 & -0.63652 & -0.06084 & -0.13916 & - \\
$4_{1}^{+}$ & 3.50 & - & 0.33134 & 1.59639 & - & - & - \\
$2_{2}^{+}$ & 3.70 & 0.04721 & -0.07248 & 0.85297 & 0.16158 & 0.17590 & - \\
\hline \hline
\end{tabular}




$$
S_{u n c}\left(J_{f}^{\pi} \neq 0^{+}\right)=\frac{n(n-1)}{2}\left[\frac{2(n-2)}{(n-1)} \frac{\left(2 J_{f}+1\right)}{(2 j-1)(2 j+1)}\right] \text {, }
$$

with $\sum_{J_{f}} S_{u n c}\left(J_{f}^{\pi}\right)=n(n-1) / 2$. This yields $S_{u n c}\left(0^{+}\right)=4 / 3$, $S_{u n c}\left(2^{+}\right)=5 / 3$, and $S_{u n c}\left(4^{+}\right)=3$, with $\sum_{J_{f}} S_{u n c}\left(J_{f}^{\pi}\right)=6$ in our ${ }^{28} \mathrm{Mg}$ case, shown in Table I. This distribution fails to reproduce the pattern of the measured ${ }^{26} \mathrm{Ne}$ partial cross sections. When multiplied by the unit cross section they overestimate both the expected $\sigma\left(2^{+}\right)$and $\sigma\left(4^{+}\right)$cross sections in comparison with the measured $\sigma\left(0^{+}\right)$. Clearly the low measured cross section yield to the two $2^{+}$states presents a particular problem for this very simple model. Already at this level, these results suggest that the data are expected to reflect the presence of correlation effects.

It is clear from the two-nucleon knockout formalism of Sec. II that there is no longer a separation of the theoretical cross section into a structure (spectroscopic) factor and a unit cross section. It is nevertheless useful in comparing between calculations and data to think of cross section ratios as effective spectroscopic factors. It is useful in the case of ${ }^{28} \mathrm{Mg}$ to present these effective spectroscopic factors as the ratio of the theoretical and/or experimental cross sections to the unit (uncorrelated) pair cross section, $\sigma_{22}=0.29 \mathrm{mb}$. The experimental and theoretical spectroscopic factors $S_{\text {expt }}$ and $S_{t h}$ listed in Table I have been calculated in this way.

\section{Fully correlated calculations}

Calculations with the fully correlated proton wave functions (using the formalism described in Sec. II) are shown as $\sigma_{\text {th }}$ (in mb) in Table I. The shell model amplitudes $C_{\alpha}^{J_{i} J_{f} I}$ used in these calculations are collected in Table II. There is good agreement of the partial cross sections $\sigma_{t h}$, and hence trivially of the theoretical spectroscopic factors $S_{t h}$, with the corresponding experimental values. The calculated inclusive cross section to the four bound states is now $1.43 \mathrm{mb}$, also in good agreement with the measured inclusive cross section of $1.50(10) \mathrm{mb}$ [21]. It should be emphasized that there is no scaling or renormalization of these cross sections, which are calculated in an absolute sense. It is also clear that in this

${ }^{28} \mathrm{Mg}$ case, a significant fraction of the integrated cross section expected, based on the $\left[0 d_{5 / 2}\right]^{4}$ uncorrelated estimate, $1.8 \mathrm{mb}$, is accounted for in the measurements to the four ${ }^{26} \mathrm{Ne}$ bound states, with $\sum_{J_{f}} S_{t h}\left(J_{f}^{\pi}\right)=4.94$.

\section{PAIR CORRELATIONS}

Having performed our complete, fully correlated calculations, this section considers the importance of specific spin correlations of the removed proton pair. These considerations, focussing on the spin-singlet knockout contribution, are facilitated by expanding the two-nucleon shell model wave functions of Eq. (2) in the $L S$ representation. So, with the nucleon spin $s=1 / 2$ and $\phi_{\ell j}^{m}(\vec{r})=u_{j \ell}(r) Y_{\ell m}(\hat{\vec{r}})$ understood, and showing the isospin explicitly,

$$
\begin{aligned}
{\left[\overline{\phi_{j_{1}}(1) \otimes \phi_{j_{2}}(2)}\right]_{I \mu}^{T \tau}=} & D_{\alpha} \hat{j}_{1} \hat{j}_{2} \sum_{L S \Lambda \Sigma m_{1} m_{2}} \hat{L} \hat{S}\left(\ell_{1} m_{1} \ell_{2} m_{2} \mid L \Lambda\right)(L \Lambda S \Sigma \mid I \mu) \mathcal{X}_{S \Sigma}(1,2) \chi_{T \tau}(1,2) \\
& \times\left[\phi_{\ell_{1} j_{1}}^{m_{1}}(1) \phi_{\ell_{2} j_{2}}^{m_{2}}(2)-(-)^{S+T} \phi_{\ell_{1} j_{1}}^{m_{1}}(2) \phi_{\ell_{2} j_{2}}^{m_{2}}(1)\right]\left\{\begin{array}{lll}
\ell_{1} & S & j_{1} \\
\ell_{2} & S & j_{2} \\
L & S & I
\end{array}\right\} .
\end{aligned}
$$

It is clear that provided the $S$ matrices are spin independent then the stripping cross section will be an incoherent sum of the contributions from the $S=0$ and $S=1$ spin components.

\section{A. Cluster removal approximations}

It should be recalled that in the $(T=1)$-pair two-nucleon transfer reactions, the dominant pieces of both the $\left\langle\left. n\right|^{3} \mathrm{He}\right\rangle$ and $\left\langle\left. p\right|^{3} \mathrm{H}\right\rangle$ projectile structure vertices preferentially select ${ }^{(2 S+1)} \ell_{J}={ }^{1} s_{0}$ spin-singlet, relative $s$-state pairs [33]. No such structure selection rule operates in the inclusive pair absorption (stripping) expression, Eq. (5). There are nevertheless some less specific (spatial) correlations driven by the reaction mechanism. It is expected, for instance, that the fast peripheral reaction will favor those configurations in which there are significant amplitudes for finding two nucleons on the same side of the projectile. The extent to which the knockout mechanism probes features of the wave function distinct from the singlet $s$-wave correlations of two-nucleon transfer is therefore of significant interest.

Motivated by this (historical) ${ }^{1} s_{0}, T=1$ transfer reaction selectivity, in [21] an estimate was made of these relative spectroscopic strengths using the methods of [34]. These are reproduced as $S_{r e l}\left(J_{f}^{\pi}\right)$ in Table III, normalized to $S_{\text {expt }}\left(4^{+}\right)$. Very similar (approximate) strengths are achieved by an alternative scheme, retaining the ${ }^{1} s_{0}$ component of each shell model two-proton configuration by constructing the linear combination of amplitudes 
TABLE III. Calculated and experimental cross sections and effective spectroscopic factors for two-proton knockout from ${ }^{28} \mathrm{Mg}$ at 82.3 MeV/nucleon. In addition to the uncorrelated $S_{\text {unc }}$, the theoretical spectroscopic factors are calculated approximately assuming ${ }^{1} s_{0}$ two-proton relative motion (for $S_{r e l}$ and $S_{r e l}^{\prime}$ ) and when including the $S=0$ configurations exactly (for $S_{S=0}$ and $\sigma_{S=0}$ ). The bracketed terms indicate relative spectroscopic factors that have been normalized to the experimental $4^{+}$state value.

\begin{tabular}{ccccccccc}
\hline \hline$J_{f}^{\pi}$ & $S_{\text {unc }}$ & $S_{\text {rel }}$ & $S_{\text {rel }}^{\prime}$ & $S_{S=0}$ & $S_{\text {exp }}$ & $S_{\text {th }}$ & $\sigma_{\text {th }}(\mathrm{mb})$ & $\sigma_{S=0}(\mathrm{mb})$ \\
\hline $0^{+}$ & 1.33 & 1.6 & 1.88 & 3.70 & $2.4(5)$ & 1.83 & 0.532 & 0.484 \\
$2^{+}$ & 1.67 & 0.14 & 0.15 & 0.26 & $0.3(5)$ & 0.54 & 0.157 & 0.034 \\
$4^{+}$ & 3.00 & $(2.0)$ & $(2.0)$ & $(2.0)$ & $2.0(3)$ & 1.79 & 0.518 & 0.259 \\
$2_{2}^{+}$ & - & 0.46 & 0.43 & 0.95 & $0.5(3)$ & 0.78 & 0.225 & 0.123 \\
\hline \hline
\end{tabular}

$\beta\left(J_{f}\right)=\sum_{\alpha} \hat{j}_{1} \hat{j}_{2} \hat{J}_{f} C_{\alpha}^{0 J_{f} J_{f}}\left\langle 00, N J_{f} ; J_{f} \mid n_{1} \ell_{1}, n_{2} \ell_{2} ; J_{f}\right\rangle\left\{\begin{array}{ccc}\ell_{1} & s & j_{1} \\ \ell_{2} & s & j_{2} \\ J_{f} & 0 & J_{f}\end{array}\right\}$.

Here $\left\langle 00, N J_{f} ; J_{f} \mid n_{1} \ell_{1}, n_{2} \ell_{2} ; J_{f}\right\rangle$ is the Moshinsky bracket for projecting the $0 s$-relative motion of the two protons from assumed oscillator single-particle wave functions [35]. These calculated (relative) spectroscopic strengths, $S_{\text {rel }}^{\prime}\left(J_{f}\right)$ $=\left|\beta\left(J_{f}\right)\right|^{2}$, are also shown in Table III, once again normalized to the $S_{\text {expt }}\left(4^{+}\right)$. These relative strengths suggest suppressed $2^{+}$state contributions, as required by the data, but treat the different components of the two-nucleon amplitude only approximately. As we point out below, there is no need to make such approximations and the full $S=0$ pair contribution can be evaluated exactly. This evaluation and the results are presented in the next subsection.

\section{B. Singlet and triplet spin correlations}

Since the partial cross sections are an incoherent sum of contributions from the $S=0$ and $S=1$ pair components, we calculate the simpler $S=0$ contribution. We can combine several of the angular momentum factors in Eq. (19) with the amplitudes $C_{\alpha}$ (where $S=0$, and hence $L=I$ will be taken), as

$$
\overline{\mathcal{C}}_{\alpha, S}^{J_{i} J_{f} I}=C_{\alpha}^{J_{i} J_{f}} \hat{j}_{1} \hat{j}_{2} \hat{L} \hat{S}\left\{\begin{array}{llc}
\ell_{1} & s & j_{1} \\
\ell_{2} & s & j_{2} \\
L & S & I
\end{array}\right\} .
$$

Hence,

$$
\begin{aligned}
{\left[C_{\alpha}^{J_{j} J_{f}}[\right.} & \left.\left.\overline{\phi_{j_{1}}(1) \otimes \phi_{j_{2}}(2)}\right]_{I \mu}^{T \tau}\right]_{S=0} \\
= & \overline{\mathcal{C}}_{\alpha, 0}^{J_{j} J_{f} I} D_{\alpha} \sum_{m_{1} m_{2}}\left(\ell_{1} m_{1} \ell_{2} m_{2} \mid I \mu\right) \\
& \times \mathcal{X}_{00}(1,2) \chi_{T \tau}(1,2) \times\left[\phi_{\ell_{1} j_{1}}^{m_{1}}(1) \phi_{\ell_{2} j_{2}}^{m_{2}}(2)\right. \\
& \left.-(-)^{T} \phi_{\ell_{1} j_{1}}^{m_{1}}(2) \phi_{\ell_{2} j_{2}}^{m_{2}}(1)\right]
\end{aligned}
$$

and the $S=0$ component of Eq. (11) is then

$$
\begin{gathered}
\frac{1}{\hat{J}_{i}^{2}} \sum_{M_{i}}\left\langle\Psi_{J_{i} M_{i}}^{(f)}\left|\left(1-\left|\mathcal{S}_{1}\right|^{2}\right)\left(1-\left|\mathcal{S}_{2}\right|^{2}\right)\right| \Psi_{J_{i} M_{i}}^{(f)}\right\rangle_{S=0} \\
=\sum_{\alpha \alpha^{\prime} I} 2 D_{\alpha} D_{\alpha^{\prime}} \overline{\mathcal{C}}_{\alpha^{\prime}, 0}^{J_{j} J_{f} I^{I} \overline{\mathcal{C}}_{\alpha, 0}^{J_{j} J_{f}} \hat{\ell}_{1} \hat{\ell}_{2}} \\
\quad \times \sum_{K Q} \frac{(-)^{Q}}{\hat{K}^{2}}\left[\text { direct }^{\prime}-\text { exchange' }\right] .
\end{gathered}
$$

The direct and exchange contributions are now

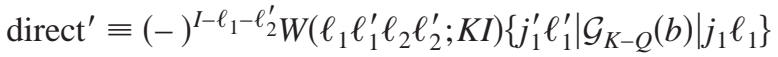

$$
\begin{aligned}
& \times\left\{j_{2}^{\prime} \ell_{2}^{\prime}\left|\mathcal{G}_{K Q}(b)\right| j_{2} \ell_{2}\right\},
\end{aligned}
$$

$$
\begin{aligned}
\text { exchange' }^{\prime} \equiv & (-)^{T+\ell_{2}^{\prime}-\ell_{1}} W\left(\ell_{1} \ell_{2}^{\prime} \ell_{2} \ell_{1}^{\prime} ; K I\right)\left\{j_{2}^{\prime} \ell_{2}^{\prime}\left|\mathcal{G}_{K-Q}(b)\right| j_{1} \ell_{1}\right\} \\
& \times\left\{j_{1}^{\prime} \ell_{1}^{\prime}\left|\mathcal{G}_{K Q}(b)\right| j_{2} \ell_{2}\right\} .
\end{aligned}
$$

Similarly to the earlier derivation, in this equation the factors $\left\{j^{\prime} \ell^{\prime}\left|\mathcal{G}_{k q}(b)\right| j \ell\right\}$ are defined such that

$$
\begin{aligned}
\int d \vec{r}\left(1-|\mathcal{S}|^{2}\right) \phi_{\ell^{\prime} j^{\prime}}^{* m^{\prime}}(\vec{r}) \phi_{\ell j}^{m}(\vec{r})= & \sum_{k q}\left(\ell^{\prime} m^{\prime} k q \mid \ell m\right) \int d \vec{r}\left(1-|\mathcal{S}|^{2}\right) \\
& \times\left\langle\left\langle j^{\prime} \ell^{\prime}\left|\mathcal{P}_{k q}(\vec{r})\right| j \ell\right\rangle\right\rangle \\
\equiv & \sum_{k q}\left(\ell^{\prime} m^{\prime} k q \mid \ell m\right) \\
& \times\left\{j^{\prime} \ell^{\prime}\left|\mathcal{G}_{k q}(b)\right| j \ell\right\}
\end{aligned}
$$

which leads to the simplified multipole terms

$$
\begin{aligned}
\left\langle\left\langle j^{\prime} \ell^{\prime}\left|\mathcal{P}_{k q}(\vec{r})\right| j \ell\right\rangle\right\rangle= & \frac{\hat{\ell}^{\prime}}{\sqrt{4 \pi}}(-1)^{\ell^{\prime}}\left(\ell 0 \ell^{\prime} 0 \mid k 0\right) u_{j^{\prime} \ell^{\prime}}(r) \\
& \times u_{j \ell}(r) Y_{k q}(\hat{\vec{r}}) .
\end{aligned}
$$

We note that the dependence on the $j$ labels remains only through the nucleon radial wave functions $u_{j \ell}(r)$.

The calculated $S=0$ cross section components $\sigma_{S=0}\left(J_{f}^{\pi}\right)$ are shown in Table III, together with their associated effective spectroscopic factors, normalized to $S_{\text {expt }}\left(4^{+}\right)$for comparisons with the approximate $S_{r e l}$ and $S_{r e l}^{\prime}$. It is clear, however, in comparison with the fully correlated calculations, that the full partial cross sections receive considerable contributions also from triplet-spin configurations to a statedependent extent. This result is extremely interesting, suggesting that the two-nucleon knockout reaction can be used to probe the spin content of the wave functions of manybody structure theories and having distinctive signatures in the different $J_{f}^{\pi}$ final states.

To reinforce this point, it is interesting to note that within our $\left[0 d_{5 / 2}\right]^{4}$ uncorrelated estimate of Sec. III B, whence $\Sigma_{J_{f}} S_{\text {unc }}\left(J_{f}^{\pi}\right)=6$, the corresponding predicted summed strengths, assuming the $S=0$ amplitudes only, would be 2.2 . The observed integrated strength of 5.2(4), Table I, and the calculated (fully correlated) strength of 4.94 clarify the importance of knockout of triplet-spin, $T=1$ nucleon pairs. 


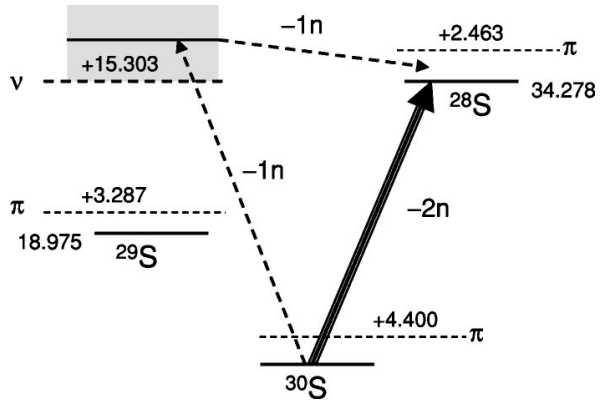

FIG. 3. The analog of Fig. 2 for the neutron-deficient ${ }^{30,29,28} \mathrm{~S}$ isotopes, showing the single-neutron $(\nu)$ and proton $(\pi)$ separation energies for each nucleus. The diagram shows that nondirect twoneutron removal to bound states in ${ }^{28} \mathrm{~S}$, by one-neutron removal to excited ${ }^{29} \mathrm{~S}$ and then neutron evaporation, would involve states far above the proton evaporation threshold and is expected to be negligible.

\section{TWO-NEUTRON KNOCKOUT}

It should be clear that the methods discussed here are applicable throughout the nuclear chart. However, as has been clarified, only in those cases where indirect paths for two-nucleon knockout to the final states are ineffective, will the stripping term considered here be expected to provide a quantitative description of measured cross sections. Such a situation also exists for two-neutron knockout from nuclei situated on the neutron-deficient side of the line of stability. The reaction thresholds in the case of ${ }^{30} \mathrm{~S}$ are shown in Fig. 3. It is fully expected that the two-neutron knockout reaction would be direct in nature in this and similar cases. The appropriate energy thresholds in the cases of the ${ }^{34} \mathrm{Ar}$ and ${ }^{26} \mathrm{Si}$ projectile systems are very similar. These three examples are calculated and presented in detail below to display more generally the sensitivity of the calculated cross sections to the shell model structure and spectroscopy.

The theoretical inputs are essentially the same as for the earlier Mg two-proton knockout case except that the twoneutron separation energies for the calculation of the single particle states are now $S_{2 n}=34.07,34.28$, and $32.34 \mathrm{MeV}$ for
${ }^{26} \mathrm{Si},{ }^{30} \mathrm{~S}$, and ${ }^{34} \mathrm{Ar}$, respectively. The rms matter radii of the (assumed Gaussian) A-body residues, for the calculation of the $S$ matrices, are 3.07, 3.17, and $3.26 \mathrm{fm}$. The calculations were carried out at $70 \mathrm{MeV} /$ nucleon. To reveal some details of the structural sensitivity of our calculations, the fully correlated stripping cross sections to the first eight shell model states of each residue are presented in Table IV. It is understood, however, that in all three cases only the $0_{1}^{+}$and $2_{1}^{+}$ residue final states are bound and so would be expected to be populated in an experiment.

Table IV shows the very significant extent to which correlations, and the details of the two-nucleon amplitudes, affect the calculated $J_{f}^{\pi}$ state cross sections. Examples of this are the relative magnitudes of the cross sections to the $0_{1}^{+}$and $\mathrm{O}_{2}^{+}$states in the different systems, and also the magnitudes of the calculated cross sections to the $2_{2}^{+}$and $2_{3}^{+}$states between the three different systems.

The simplest (uncorrelated) estimates for these systems can also be carried out, as was discussed in Sec. III B. For ${ }^{30} \mathrm{~S}$, for example, assuming a $\left[0 d_{5 / 2}\right]^{6}$ ground state, then $\sum_{J_{f}} S_{\text {unc }}\left(J_{f}^{\pi}\right)=n(n-1) / 2=15$, with spectroscopic factors $S_{\text {unc }}\left(0^{+}\right)=1, S_{\text {unc }}\left(2^{+}\right)=5$, and $S_{\text {unc }}\left(4^{+}\right)=9$. The corresponding calculated unit cross section is now $\sigma_{22}=0.162 \mathrm{mb}$, suggesting an integrated cross section of order $15 \times \sigma_{22} \approx 2.43 \mathrm{mb}$, and that $\sigma\left(2^{+}\right) \approx 5 \sigma\left(0^{+}\right)$. As we see above, in ${ }^{30} \mathrm{~S}$ the calculated bound-final-state cross sections are only $\sigma_{\text {th }}\left(0_{1}^{+}\right)$ $=0.27 \mathrm{mb}$ and $\sigma_{\text {th }}\left(2_{1}^{+}\right)=0.22 \mathrm{mb}$, with an expected inclusive cross section of only $0.50 \mathrm{mb}$, and with $\sigma_{t h}\left(2_{1}^{+}\right) \approx \sigma_{t h}\left(0_{1}^{+}\right)$. This observation and the detailed analyses shown on Table IV confirm that the simple, uncorrelated nucleon-pair predictions will, in general, be rather poor and should be used with some caution. Unlike for the earlier ${ }^{28} \mathrm{Mg}$ example, here the total two-nucleon stripping strength is distributed over a large number of residue final state transitions, most of which are above the proton thresholds of the final states.

\section{SUMMARY AND CONCLUSIONS}

A comprehensive scheme is presented for the calculation of the cross sections for two-nucleon knockout by the inelas-

TABLE IV. Calculated stripping cross sections for two-neutron knockout from ${ }^{34} \mathrm{Ar},{ }^{30} \mathrm{~S}$, and ${ }^{26} \mathrm{Si}$ at $70 \mathrm{MeV} /$ nucleon. Calculations for transitions leading the lowest eight shell model $J_{f}^{\pi}$ configurations of the ${ }^{32} \mathrm{Ar},{ }^{28} \mathrm{~S}$, and ${ }^{24} \mathrm{Si}$ nuclei are shown.

\begin{tabular}{ccccccccc}
\hline \hline & ${ }^{26} \mathrm{Si} \rightarrow{ }^{24} \mathrm{Si}\left(J_{f}^{\pi}\right)$ & \multicolumn{3}{c}{${ }^{30} \mathrm{~S} \rightarrow{ }^{28} \mathrm{~S}\left(J_{f}^{\pi}\right)$} & \multicolumn{3}{c}{${ }^{34} \mathrm{Ar} \rightarrow{ }^{32} \operatorname{Ar}\left(J_{f}^{\pi}\right)$} \\
\hline$J_{f}^{\pi}$ & $\begin{array}{c}E^{*} \\
(\mathrm{MeV})\end{array}$ & $\begin{array}{c}\sigma_{\text {th }} \\
(\mathrm{mb})\end{array}$ & $J_{f}^{\pi}$ & $\begin{array}{c}E^{*} \\
(\mathrm{MeV})\end{array}$ & $\begin{array}{c}\sigma_{\text {th }} \\
(\mathrm{mb})\end{array}$ & $J_{f}^{\pi}$ & $\begin{array}{c}E^{*} \\
(\mathrm{MeV})\end{array}$ & $\begin{array}{c}\sigma_{\text {th }} \\
(\mathrm{mb})\end{array}$ \\
\hline $0_{1}^{+}$ & 0.0 & 0.427 & $0_{1}^{+}$ & 0.0 & 0.273 & $0_{1}^{+}$ & 0.0 & 0.260 \\
$2_{1}^{+}$ & 2.15 & 0.105 & $2_{1}^{+}$ & 1.54 & 0.223 & $2_{1}^{+}$ & 2.09 & 0.109 \\
$2_{2}^{+}$ & 3.74 & 0.124 & $0_{2}^{+}$ & 3.80 & 0.030 & $2_{2}^{+}$ & 4.21 & 0.095 \\
$4_{1}^{+}$ & 4.00 & 0.314 & $4_{1}^{+}$ & 4.13 & 0.187 & $0_{2}^{+}$ & 4.81 & 0.017 \\
$3_{1}^{+}$ & 4.57 & 0.000 & $2_{2}^{+}$ & 4.26 & 0.213 & $1_{1}^{+}$ & 5.58 & 0.002 \\
$0_{2}^{+}$ & 4.66 & 0.002 & $1_{1}^{+}$ & 4.40 & 0.002 & $3_{1}^{+}$ & 5.61 & 0.281 \\
$2_{3}^{+}$ & 5.34 & 0.101 & $2_{3}^{+}$ & 4.78 & 0.039 & $2_{3}^{+}$ & 5.65 & 0.358 \\
$4_{2}^{+}$ & 5.65 & 0.001 & $4_{2}^{+}$ & 5.19 & 0.457 & $0_{3}^{+}$ & 5.76 & 0.050 \\
\hline \hline
\end{tabular}


tic breakup or stripping mechanism. The framework is able to combine fully the shell model structure information with eikonal reaction theory. As an example of its application, we have investigated in detail the direct two-proton knockout reaction from the neutron rich nucleus ${ }^{28} \mathrm{Mg}$. We have presented the predictions of several approximations to the structure and reaction which neglect, to differing extents, the spatial and angular momentum correlations present in the full two-nucleon configuration set. The agreement of our most complete results, which use the full shell model two-nucleon amplitudes, and the experimental data is good. There is a considerable improvement in our results compared to simple no-correlation or assumed cluster-correlated approximations. We show also that the reaction is sensitive to two-proton components in the wave function other than the $S=0, T=1$ configurations probed in two-nucleon transfers, such as $(p, t)$ and $\left({ }^{3} \mathrm{He}, n\right)$. Our calculations provide considerable further evidence that the measured two-proton knockout from a neutron-rich nucleus occurs as a direct reaction, thus opening the possibility of both producing and performing detailed two-nucleon spectroscopy on the most extreme of neutronrich, or in the case of two-neutron knockout, proton-rich nuclei. The technique offers considerable promise for interrogation of two-body correlations within many-body calculations in these regions.

\section{ACKNOWLEDGMENTS}

This work was supported by the U.K. Engineering and Physical Sciences Research Council (EPSRC) under Grant No. GR/M82141 and by the National Science Foundation under Grants No. PHY-0110253, No. PHY-9875122, No. PHY-0244453, and No. PHY-0342281.
[1] A. Navin et al., Phys. Rev. Lett. 81, 5089 (1998).

[2] P. G. Hansen and B. M. Sherrill, Nucl. Phys. A693, 133 (2001).

[3] P. G. Hansen and J. A. Tostevin, Annu. Rev. Nucl. Part. Sci. 53, 219 (2003).

[4] D. Cortina-Gil et al., Phys. Lett. B 529, 36 (2002).

[5] T. Kobayashi et al., Phys. Rev. Lett. 60, 2599 (1988).

[6] E. Sauvan et al., Phys. Lett. B 491, 1 (2000).

[7] E. Sauvan et al., Phys. Rev. C 69, 044603 (2004).

[8] T. Aumann et al., Phys. Rev. Lett. 84, 35 (2000).

[9] V. Maddalena et al., Phys. Rev. C 63, 024613 (2001).

[10] J. R. Terry et al., Phys. Rev. C 69, 054306 (2004).

[11] J. A. Tostevin, J. Phys. G 25, 735 (1999).

[12] A. Navin et al., Phys. Rev. Lett. 85, 266 (2000).

[13] J. A. Tostevin, Nucl. Phys. A682, 320c (2001).

[14] J. Enders et al., Phys. Rev. C 67, 064301 (2003).

[15] A. Gade et al., Phys. Rev. C 69, 034311 (2004).

[16] A. Gade et al., Phys. Rev. Lett. 93, 042501 (2004).

[17] B. A. Brown, P. G. Hansen, B. M. Sherrill, and J. A. Tostevin, Phys. Rev. C 65, 061601(R) (2002).

[18] J. S. Al-Khalili, J. A. Tostevin, and I. J. Thompson, Phys. Rev. C 54, 1843 (1996).

[19] H. Esbensen and G. F. Bertsch, Phys. Rev. C 64, 014608 (2001).
[20] J. A. Tostevin, D. Bazin, B. A. Brown, T. Glasmacher, P. G. Hansen, V. Maddalena, A. Navin, and B. M. Sherrill, Phys. Rev. C 66, 024607 (2002).

[21] D. Bazin et al., Phys. Rev. Lett. 91, 012501 (2003).

[22] N. K. Glendenning, Phys. Rev. 137, B102 (1965).

[23] M. Hussein and K. McVoy, Nucl. Phys. A445, 124 (1985).

[24] F. Azaiez et al., Eur. Phys. J. A 15, 93 (2002).

[25] B. V. Pritychenko et al., Phys. Lett. B 461, 322 (1999); 467, 309 (1999).

[26] O. Sorlin et al., Nucl. Phys. A685, 186c (2001).

[27] R. J. Glauber, in Lectures in Theoretical Physics, edited by W. E. Brittin (Interscience, New York, 1959), Vol. 1, p. 315.

[28] S. Kox et al., Phys. Rev. C 35, 1678 (1987).

[29] L. Ray, Phys. Rev. C 20, 1857 (1979).

[30] A. Ozawa et al., Nucl. Phys. A691, 599 (2001).

[31] B. A. Brown et al., computer code OXBASH, MSU-NSCL Report No. 524.

[32] B. A. Brown and B. H. Wildenthal, Annu. Rev. Nucl. Part. Sci. 38, 29 (1988).

[33] M. H. Macfarlane and J. P. Schiffer, in Nuclear Spectroscopy and Reactions, Part B, edited by J. Cerny (Academic Press, New York, 1974), p. 169.

[34] N. Anyas-Weiss et al., Phys. Rep., Phys. Lett. 12, 201 (1974).

[35] M. Baranger and K. T. R. Davies, Nucl. Phys. 79, 403 (1966). 